Proc. 13th International School on Theoretical Physics: Symmetry and Structural Properties of Condensed Matter

\title{
Three-Diagonal Blocks of Two-Magnon Hamiltonians with Specified Wave Numbers for Heisenberg Rings
}

\author{
M. ŁABUZ ${ }^{a, *}$ AND J. MILEWSKI ${ }^{b}$ \\ ${ }^{a}$ Department of Theoretical Physics, Faculty of Mathematics and Natural Sciences, \\ University of Rzeszów, S. Pigonia 1, 35-310 Rzeszów, Poland \\ ${ }^{b}$ Institute of Mathematics, Faculty of Electrical Engineering, \\ Poznań University of Technology, Piotrowo 3A, 60-965 Poznań, Poland
}

\begin{abstract}
We demonstrate the method of creating blocks of Hamiltonian matrices for Heisenberg rings with $N$ nodes and $r=2$ overturned spins, depending on total quasimomentum $k$. Initial problem of dimension $\left(\begin{array}{c}N \\ 2\end{array}\right)$ reduces, approximately, $N$-tuply, depending on $N$ and $k$ numbers. We consider block Hamiltonians using a particular basis, called wavelet basis. In this basis, these blocks take three-diagonal form.
\end{abstract}

DOI: 10.12693/APhysPolA.135.1255

PACS/topics: 75.10.Jm, 03.65.Fd, 02.10.Ud, 02.10.De

\section{Introduction}

In 1931 Hans Bethe introduced his famous ansatz concerning the diagonalization of the Heisenberg Hamiltonian of a ring consisting of $N$ nodes [1]. However, Bethe ansatz equations has turned out to be a very complicated set of nonlinear algebraic equations. To solve such a set one applies sophisticated algebraic, and combinatoric methods, including spectral parameters, rigged string configurations, etc. [2-7]. As matrix elements of the Heisenberg Hamiltonian are of the arithmetic form [8], solutions of the eigenproblem reveal the Galois symmetry [9-11].

It is well known that sectors with a given number of magnons are invariant with respect to the Heisenberg Hamiltonian $\hat{H}$. Moreover, the subspaces with a given wave number $k$ are also $\hat{H}$-invariant. The Hilbert space $\mathcal{H}$ of all quantum states can be decomposed, with respect to $r$ and $k$, in the following way:

$$
\mathcal{H}=\bigoplus_{r, k} \mathcal{H}_{r, k} \text {. }
$$

This decomposition is compatible with the decomposition of the Hamiltonian $\hat{H}$ into blocks

$$
H=\bigoplus H_{r, k} .
$$

We consider here the two-magnon sector $(r=2)$ for the Heisenberg ring with $N$ nodes. Geometry of a ring, as well as the Heisenberg Hamiltonian, displays the symmetry of the cyclic group $\mathbb{C}_{N}$, isomorphic with $\mathbb{Z}_{N}$ group, and even richer $\mathbb{D}_{N}$ group. In this paper we focus on the $\mathbb{C}_{N}$ subgroup, as it enables to describe the basis of orbits and the Fourier transform from the basis of orbits to the basis of wavelets.

*corresponding author; e-mail: labuz@ur.edu.pl
The classical configuration space for the system being discussed has been considered e.g. in [12]. In this paper we deal with the space of quantum states. In particular, we present matrices of block Hamiltonians $H_{2, k}$ with respect to $k$ and $N$, including their parity, in the so-called wavelet basis $[10,13,14]$. Especially, the eigenproblem of the system being considered has been studied in [13] by means of the Chebyshev polynomials. The paper is organized as follows. First, we introduce basis of positions, orbits, and wavelets as well. Next, we consider the action of the Hamiltonian on basis vectors of the auxiliary basis of orbits, and, eventually, in the basis of wavelets. In this way we get consecutive columns of the block Hamiltonian matrices. As a result of such a procedure one gets Hamiltonian matrices of the three-diagonal form.

\section{Bases}

\subsection{Basis of positions}

A magnetic configuration is described by positions of nodes with overturned spins. In particular, to define a magnetic configuration with $r$ spin deviations, one has to give an $r$-element set of all positions of deviations. Any magnetic configuration for two spin deviations is of the form

$$
\left\{j_{1}, j_{2}\right\}: j_{1}, j_{2} \in \mathbb{Z}_{N}, \quad j_{1} \neq j_{2} .
$$

Notice that the brace in (3) denotes a set, and not a sequence, so that the order within a configuration $\left\{j_{1}, j_{2}\right\}$ is irrelevant.

With configurations described in (3) there is a related basis of positions, indexed by

$$
\boldsymbol{j}=\left|\left\{j_{1}, j_{2}\right\}\right\rangle, \quad 1 \leq j_{1}<j_{2} \leq N,
$$

which, by definition, is an orthonormal basis, so that it determines explicitly the scalar product in this space. The range of $j_{1}, j_{2}$ in (4) is the so-called Yang-Baxter condition. It is worth to observe that sets $\left\{j_{1}, j_{2}\right\}$ in (3) are doubly repeating, while condition (4) eliminates this problem. 
Remark. It is worth to observe that the basis of positions (4) in quantum information theory is called computational basis (for the case of two spin deviations). Naturally, analogous nomenclature takes place for the other case of number of spin deviations.

\subsection{Basis of orbits}

Elements of basis of orbits are labeled by one of the number of nodes, and by the vector of relative positions of nodes, so that in the case of two magnons it can be introduced as

$$
\left|j, \boldsymbol{t}_{\alpha}\right\rangle=|\{j, j+\alpha\}\rangle, \quad \alpha=1,2, \ldots,\left[\frac{N}{2}\right],
$$

where $\boldsymbol{t}_{\alpha}=(\alpha, N-\alpha)$ is the vector of the relative configuration, which defines the orbit, with $\alpha$ indicating the distance between two spin deviations in a configuration. In (5), $j \in \mathbb{Z}_{N}$, except of even $N$ and $\alpha=\frac{N}{2}$, when $j=1, \ldots, \frac{N}{2}$, which results from the fact that the orbit in this particular case is rarefied. Note that the limitation of variability of $j$ for $\alpha=\frac{N}{2}$, and even $N$, similar to the case of the Yang-Baxter condition, is imposed to avoid the repetition of basis vectors. The basis of orbits is a permuted basis of positions (cf. (5)) - all basis vectors are the same; the only difference arises in the way of numeration. Such a change enables to numerate basis vectors according to orbits. In detail, $\boldsymbol{t}_{\alpha}$ is an invariant of the action of the group of translation and precisely determines the orbit $\left(\boldsymbol{t}_{\alpha}\right.$ in (5) corresponds to the orbit and $j$ is a number of element in the orbit). By acting the group of translation on $\left|j, t_{\alpha}\right\rangle$ vectors there is only a change of the number of an element in an orbit, while $\boldsymbol{t}_{\alpha}$ remains unchanged, which highlights the concept of the basis of orbits. However, by acting on the basis of positions there is a simultaneous change of $j_{1}$ and $j_{2}$. As it results from (5), $\left|j, \boldsymbol{t}_{\alpha}\right\rangle$ shows that the transition from one basis to the another is a permutation.

\subsection{Basis of wavelets}

Basis of wavelets can be formed by a single, discrete Fourier transform with respect to the first index of the basis of orbits $j$ :

$$
\left|\boldsymbol{t}_{\alpha}, k\right\rangle=\frac{1}{\sqrt{N}} \sum_{j=1}^{N} \omega^{-j k}\left|j, \boldsymbol{t}_{\alpha}\right\rangle, \text { for } \alpha \neq \frac{N}{2},
$$

and

$$
\begin{aligned}
\left|\boldsymbol{t}_{\alpha}, k\right\rangle & =\sqrt{\frac{2}{N}} \sum_{j=1}^{\frac{N}{2}} \omega^{-j k}\left|j, \boldsymbol{t}_{\alpha}\right\rangle, \\
\text { for } \alpha & =\frac{N}{2}, \text { and } N \text { even, only for } k \text { even, }
\end{aligned}
$$

with admissible quasimomenta $k$ from the range

$$
k=0, \pm 1, \pm 2, \ldots, \begin{cases} \pm(N / 2-1), N / 2 & \text { for } \mathrm{N} \text { even }, \\ \pm(N-1) / 2 & \text { for } \mathrm{N} \text { odd. }\end{cases}
$$

The matter of different coefficients and summation range in $(7)$ is related with a rarefied orbit in comparison with a regular orbit in (6).
Remark. It is a very important fact that for the case of $k$ odd, $\alpha=\frac{N}{2}$ and $N$ even, $\boldsymbol{t}_{\alpha}$ and $k$ do not fit within the range of indices of the basis of wavelets.

\section{The action of blocks of Hamiltonians in different bases for two magnons}

A general form of the Hamiltonian for $r=2$ spin deviations in the space $\mathcal{H}$ can be introduced as

$$
\hat{H}|\boldsymbol{j}\rangle=\sum_{\boldsymbol{j}^{\prime}}\left(\left|\boldsymbol{j}^{\prime}\right\rangle-|\boldsymbol{j}\rangle\right),
$$

where summation runs over the nearest neighbour configurations $\boldsymbol{j}^{\prime}$ of $\boldsymbol{j}$.

It is worth to notice that the choice of the sign of the Hamiltonian corresponds to the case of the antiferromagnetic ring. The change of the sign leads to the ferromagnetic model.

\subsection{The Hamiltonian in the basis of positions}

In details, in the case of the basis of positions, the Hamiltonian can be introduced in the following way:

$$
\begin{aligned}
& \hat{H}|\{j, j+1\}\rangle=|\{j-1, j+1\}\rangle+|\{j, j+2\}\rangle \\
& \quad-2|\{j, j+1\}\rangle, \\
& \hat{H}\left|\left\{j, j^{\prime}\right\}\right\rangle=\left|\left\{j-1, j^{\prime}\right\}\right\rangle+\left|\left\{j+1, j^{\prime}\right\}\right\rangle+\left|\left\{j, j^{\prime}-1\right\}\right\rangle \\
& \quad+\left|\left\{j, j^{\prime}+1\right\}\right\rangle-4|\{j, j+1\}\rangle, \text { for } d\left(j, j^{\prime}\right)>1 .
\end{aligned}
$$

\subsection{The Hamiltonian in the basis of orbits}

Now, recall that the basis of orbits is the permuted basis of positions, depending on the distance $\alpha$ and the parity of $N$, hence, the Hamiltonian operates on vectors of this basis in the following way:

$$
\text { - } \alpha=1
$$

$$
\hat{H}\left|j, \boldsymbol{t}_{\alpha}\right\rangle=\left|j-1, \boldsymbol{t}_{\alpha+1}\right\rangle+\left|j, \boldsymbol{t}_{\alpha+1}\right\rangle-2\left|j, \boldsymbol{t}_{\alpha}\right\rangle,
$$

- $\alpha=2,3, \ldots,\left[\frac{N}{2}\right]-1$ for $N$ odd; $\alpha=2,3, \ldots, \frac{N}{2}-2$ for $N$ even

$$
\begin{gathered}
\hat{H}\left|j, \boldsymbol{t}_{\alpha}\right\rangle=\left|j-1, \boldsymbol{t}_{\alpha+1}\right\rangle+\left|j+1, \boldsymbol{t}_{\alpha-1}\right\rangle \\
+\left|j, \boldsymbol{t}_{\alpha-1}\right\rangle+\left|j, \boldsymbol{t}_{\alpha+1}\right\rangle-4\left|j, \boldsymbol{t}_{\alpha}\right\rangle,
\end{gathered}
$$

- $\alpha=\left[\frac{N}{2}\right]$ for $N$ odd

$$
\begin{gathered}
\hat{H}\left|j, \boldsymbol{t}_{\alpha}\right\rangle=\left|j+\boldsymbol{t}_{\alpha}, \boldsymbol{t}_{\alpha}\right\rangle+\left|j+\boldsymbol{t}_{\alpha+1}, \boldsymbol{t}_{\alpha}\right\rangle \\
+\left|j, \boldsymbol{t}_{\alpha-1}\right\rangle+\left|j+1, \boldsymbol{t}_{\alpha-1}\right\rangle-4\left|j, \boldsymbol{t}_{\alpha}\right\rangle
\end{gathered}
$$

- $\alpha=\frac{N}{2}$ for $N$ even

$$
\begin{aligned}
& \hat{H}\left|j, \boldsymbol{t}_{\alpha}\right\rangle=\left|j+\boldsymbol{t}_{\alpha}, \boldsymbol{t}_{\alpha-1}\right\rangle+\left|j+1, \boldsymbol{t}_{\alpha-1}\right\rangle \\
& \quad+\left|j, \boldsymbol{t}_{\alpha-1}\right\rangle+\left|j+\boldsymbol{t}_{\alpha+1}, \boldsymbol{t}_{\alpha-1}\right\rangle-4\left|j, \boldsymbol{t}_{\alpha}\right\rangle .
\end{aligned}
$$




\subsection{The Hamiltonian in the basis of wavelets}

Now, keeping in mind that the basis of wavelets is a single Fourier transform with respect to $j$, within a one orbit, taking into account the distance $\alpha$ and the parity of $N$ and $k$ (if necessary, shifting the summand variable $j$ in (12)-(15)), the action of the Hamiltonian in this basis can be presented as

- $\alpha=1$

$$
\hat{H}\left|\boldsymbol{t}_{\alpha}, k\right\rangle=\left(1+\omega^{-k}\right)\left|\boldsymbol{t}_{\alpha+1}, k\right\rangle-2\left|\boldsymbol{t}_{\alpha}, k\right\rangle,
$$

- $\alpha=2,3, \ldots,\left[\frac{N}{2}\right]-1$ for $N$ odd; $\alpha=2,3, \ldots, \frac{N}{2}-2$ for $N$ even

$$
\begin{aligned}
& \hat{H}\left|\boldsymbol{t}_{\alpha}, k\right\rangle=\left(1+\omega^{k}\right)\left|\boldsymbol{t}_{\alpha-1}, k\right\rangle \\
& \quad+\left(1+\omega^{-k}\right)\left|\boldsymbol{t}_{\alpha+1}, k\right\rangle-4\left|\boldsymbol{t}_{\alpha}, k\right\rangle,
\end{aligned}
$$

- $\alpha=\left[\frac{N}{2}\right]$ for $N$ odd

$$
\begin{aligned}
& \hat{H}\left|\boldsymbol{t}_{\alpha}, k\right\rangle=\left(1+\omega^{k}\right)\left|\boldsymbol{t}_{\alpha-1}, k\right\rangle \\
& \quad+\left(\omega^{k \boldsymbol{t}_{\alpha}}+\omega^{(k+1) \boldsymbol{t}_{\alpha}}\right)\left|\boldsymbol{t}_{\alpha}, k\right\rangle-4\left|\boldsymbol{t}_{\alpha}, k\right\rangle,
\end{aligned}
$$

- $\alpha=\frac{N}{2}-1$ for $N$ even, $k$ even

$$
\begin{aligned}
& \hat{H}\left|\boldsymbol{t}_{\alpha}, k\right\rangle=\left(1+\omega^{k}\right)\left|\boldsymbol{t}_{\alpha-1}, k\right\rangle \\
& \quad+\sqrt{2}\left(1+\omega^{-k}\right)\left|\boldsymbol{t}_{\alpha+1}, k\right\rangle-4\left|\boldsymbol{t}_{\alpha}, k\right\rangle
\end{aligned}
$$

- $\alpha=\frac{N}{2}$ for $N$ even, $k$ even

$$
\begin{gathered}
\hat{H}\left|\boldsymbol{t}_{\alpha}, k\right\rangle=\sqrt{\frac{2}{N}} \sum_{j=1}^{\frac{N}{2}} \omega^{-k j} H\left|j, \boldsymbol{t}_{\alpha}\right\rangle= \\
\sqrt{2}\left(\omega^{k}+1\right)\left|\boldsymbol{t}_{\alpha-1}, k\right\rangle-4\left|\boldsymbol{t}_{\alpha}, k\right\rangle \\
\bullet \alpha=\frac{N}{2}-1 \text { for } N \text { even, } k \text { odd } \\
\hat{H}\left|\boldsymbol{t}_{\alpha}, k\right\rangle=\left(1+\omega^{k}\right)\left|\boldsymbol{t}_{\alpha-1}, k\right\rangle-4\left|\boldsymbol{t}_{\alpha}, k\right\rangle .
\end{gathered}
$$

\section{Hamiltonian matrices}

As a result of considerations presented above, we may introduce Hamiltonian matrices depending on the parity of $N$ and $k$. Here we put $c=1+\omega^{k}$, and $c^{*}=1+\omega^{-k}$.

- $N$ odd

$$
H=\left(\begin{array}{ccccccc}
-2 & c_{k} & 0 & \ldots & \ldots & \ldots & \ldots \\
c_{k}^{*} & -4 & c_{k} & \ldots & \ldots & \ldots & \ldots \\
0 & c_{k}^{*} & -4 & \ldots & \ldots & \ldots & \ldots \\
0 & 0 & c_{k}^{*} & \ddots & \ldots & \ldots & \ldots \\
\vdots & \vdots & \vdots & \vdots & \ddots & c_{k} & 0 \\
\vdots & \vdots & \vdots & \vdots & \ldots & -4 & c_{k} \\
\vdots & \vdots & \vdots & \vdots & \ldots & c_{k}^{*} & d_{k}-4
\end{array}\right)_{\left[\frac{N}{2}\right] \times\left[\frac{N}{2}\right]}
$$

where $d_{k}=(-1)^{k}\left(\omega^{\frac{k}{2}}+\omega^{-\frac{k}{2}}\right)$.
- $N$ even, $k$ even

$$
H=\left(\begin{array}{ccccccc}
-2 & c_{k} & 0 & \ldots & \ldots & \ldots & \ldots \\
c_{k}^{*} & -4 & c_{k} & \ldots & \ldots & \ldots & \ldots \\
0 & c_{k}^{*} & -4 & \ldots & \ldots & \ldots & \ldots \\
0 & 0 & c_{k}^{*} & \ddots & \ldots & \ldots & \ldots \\
\vdots & \vdots & \vdots & \vdots & \ddots & c_{k} & 0 \\
\vdots & \vdots & \vdots & \vdots & \vdots & -4 & \sqrt{2} c_{k} \\
\vdots & \vdots & \vdots & \vdots & \vdots & \sqrt{2} c_{k}^{*} & -4
\end{array}\right)_{\frac{N}{2} \times \frac{N}{2}}
$$

- $N$ even, $k$ odd

$$
H=\left(\begin{array}{ccccccc}
-2 & c_{k} & 0 & \ldots & \ldots & \ldots & \ldots \\
c_{k}^{*} & -4 & c_{k} & \ldots & \ldots & \ldots & \ldots \\
0 & c_{k}^{*} & -4 & \ldots & \ldots & \ldots & \ldots \\
0 & 0 & c_{k}^{*} & \ddots & \ldots & \ldots & \ldots \\
\vdots & \vdots & \vdots & \vdots & \ddots & c_{k} & 0 \\
\vdots & \vdots & \vdots & \vdots & \vdots & -4 & c_{k} \\
\vdots & \vdots & \vdots & \vdots & \vdots & c_{k}^{*} & -4
\end{array}\right)_{\left(\frac{N}{2}-1\right) \times\left(\frac{N}{2}-1\right) .}
$$

\section{The eigenproblem and Chebyshev polynomials}

Hamiltonian blocks for all cases (22)-(24) are given by means of three-diagonal matrices, and all diagonal elements, except of the first and at most the last one, are equaled to -4 . It suggests that for more convenient form of algebraic expressions for characteristic polynomials of block Hamiltonians, we shift all energy levels in these sectors by 4 . Equivalently, we consider shifted Hamiltonian in the whole 2-magnon space

$$
\hat{H}^{\prime}=\hat{H}+4 \hat{I}, \quad \hat{H}^{\prime}|\boldsymbol{j}\rangle=\sum_{\boldsymbol{j}^{\prime}}\left(\left|\boldsymbol{j}^{\prime}\right\rangle-|\boldsymbol{j}\rangle\right)+4|\boldsymbol{j}\rangle,
$$

with $\hat{I}$ being an identity operator. The summand $4 \hat{I}$ in (25) just shifts the standard energy level, but does not influence on a value of gaps between energy levels. Indeed, after such a shift, most of elements on the main diagonal of all Hamiltonian blocks vanishes.

In the theory of three-diagonal matrices, it is a well known fact that their determinants are expressed by means of the Chebyshev polynomials. The application of this theory gives the following form of the characteristic polynomial of the block Hamiltonians [13]:

$$
P_{N}^{k}(x)=h^{M} \Phi_{N, M}^{k}(-u)+2 h^{M-1} \Phi_{N, M-1}^{k}(-u),
$$
where

$$
u=\frac{x}{2 h}
$$


and

$$
\Phi_{N, n}^{k}= \begin{cases}2 T_{n} & \text { for } N, k \text { even, } \\ \frac{1}{h} U_{n-1} & \text { for } N \text { even, } k \text { odd, } \\ V_{n} & \text { for } N, k \text { odd } \\ W_{n} & \text { for } N \text { odd, } k \text { even, }\end{cases}
$$

with $M=\left[\frac{N}{2}\right]$. In the above formulae, $T_{n}, U_{n}, V_{n}$ and $W_{n}$ are the Chebyshev polynomials of the first, the second, the third, and the fourth kind, respectively. They can be defined by means of the trigonometric substitution $u=\cos \theta$ :

$$
\left\{\begin{array}{l}
T_{n}(u)=\cos n \theta, \\
U_{n}(u)=\frac{\sin (n+1) \theta}{\sin \theta}, \\
V_{n}(u)=\frac{\cos (n+1 / 2) \theta}{\cos \theta / 2}, \\
W_{n}(u)=\frac{\sin (n+1 / 2) \theta}{\sin \theta / 2} .
\end{array}\right.
$$

Moreover, $h=2 \cos (\pi k / N)$ is the modulus of hybridization parameter $c_{k}$. The characteristic equation leads to the following rational equation:

$$
R_{N}^{k}(u)=\frac{h}{2},
$$

where

$$
R_{N}^{k}(u)=-\frac{\Phi_{N, M-1}^{k}(-u)}{\Phi_{N, M}^{k}(-u)} .
$$

By choosing again the variable $x$ (27) one gets energy levels, which enables to solve the eigenproblem of Hamiltonians being considered.

\section{Conclusions}

For any number $N$ of nodes, and two spin deviations, the dimension of an eigenproblem is equal to the number of possible vectors $\boldsymbol{t}_{\alpha}$, with respect to $N$ and $k$. Similar to block Hamiltonian matrices, also secular matrices are of the three-diagonal form. In the theory of recursive sequences, there is a known fact that determinants of three-diagonal matrices satisfy linear recurrence problem of a rank 2. This feature is important and helpful for the diagonalization procedure of block Hamiltonians considered above. Indeed, characteristic polynomials are expressed by a linear combination of two adjacent Chebyshev polynomials of a given kind, depending on the parity of $N$ and $k$.

By solving Eq. (30) one may get exact energy levels. Furthermore, with the use of inverse Bethe ansatz method (e.g. [10]) one can obtain the Bethe parameters which classify eigenstates of the problem. In the case of bound states on the border of the Brillouin zone, the set of the Bethe equations is singular, and has no solutions within the complex numbers. These singularities have been studied e.g. in papers $[13,15]$ and [16].

For $r>2$ cases, one needs to apply other mathematical techniques, as the degree of complexity of the problem being discussed increases together with $r$, which is a very interesting question.

\section{References}

[1] H. Bethe, Z. Phys. 71, 205 (1931) (in German; English translation in: D.C. Mattis, The Many-Body Problem, World Sci., Singapore 1993, p. 689).

[2] S.V. Kerov, A.N. Kirillov, N.Yu. Reshetikhin, Zap. Nauchn. Sem. LOMI 155, 50 (1986) (in Russian; English translation in: J. Sov. Math. 41, 916 (1988)).

[3] L.D. Faddeev, L.A. Takhtajan, Leningr. Otd. Mat. Inst. Steklov. 109, 134 (1981) (in Russian; English translation in: J. Sov. Math. 24, 241 (1984)).

[4] F.H.L. Essler, V.E. Korepin, K. Schoutens, J. Phys. A Math. Gen. 25, 4115 (1992).

[5] W.J. Caspers, A. Wal, M. Łabuz, M. Kuźma, T. Lulek, J. Math. Phys. 45, 391 (2004).

[6] P. Jakubczyk, A. Wal, D. Jakubczyk, T. Lulek, Comp. Phys. Commun. 183, 1354 (2012).

[7] J. Milewski, B. Lulek, T. Lulek, M. Łabuz, R. Stagraczyński, Physica B 434, 14 (2014).

[8] G. Banaszak, D. Blinkiewicz, P. Krasoń, J. Milewski, Acta Phys. Pol. A 133, 441 (2018).

[9] J. Milewski, G. Banaszak, T. Lulek, M. Łabuz, R. Stagraczyński, OSID 19, 1250012 (2012).

[10] J. Milewski, G. Banaszak, T. Lulek, M. Labuz, Physica B 406, 520 (2011).

[11] G. Banaszak, B. Lulek, T. Lulek, J. Milewski, B. Szydło, Rep. Math. Phys. 71, 205 (2013).

[12] B. Lulek, D. Jakubczyk, CEJP 1, 132 (2003).

[13] J. Milewski, Rep. Math. Phys. 70, 345 (2012).

[14] M. Łabuz, J. Milewski, T. Lulek, Acta Phys. Pol. A 133, 444 (2018)

[15] R.I. Nepomechie, Ch. Wang, J. Phys. Math. A Math. Theor. 46, 325002 (2013).

[16] A. Koper, P. Krasoń, J. Milewski, Acta Phys. Pol. A 133, 438 (2018). 